\title{
Association between Carotid Artery Plaque Score and SYNTAX Score in Coronary Artery Disease Patients
}

\section{Jin-Wook Chung}

Department of Internal Medicine, Dongguk University, College of Medicine, Gyeongju, Korea

"Corresponding author: Jin-Wook Chung, Department of Cardiology, Gyeongju Hospital, Dongguk University, College of Medicine 1090-1 Suckjang dong, Gyeongju, 780-350, Republic of Korea. Tel: +82-54-770-8507; Fax: +82-54-770-8378; E-mail: jwchung75@naver.com

Rec date: September 15, 2017; Acc date: October 10, 2017; Pub date: October 16, 2017

Copyright: $\odot 2017$ Chung JW. This is an open-access article distributed under the terms of the Creative Commons Attribution License, which permits unrestricted use, distribution, and reproduction in any medium, provided the original author and source are credited.

\begin{abstract}
Background: Numerous studies have been published the correlation between plaque score (PS), mean carotid artery intima-media thickness (IMT), and the prevalence or complexity of coronary artery lesions in coronary artery disease (CAD) patients. However, very few studies have reported the association between the complexity of coronary artery lesions and PS and the mean carotid artery IMT. We therefore aimed to examine the correlation between carotid artery ultrasound parameters and complexity of coronary artery lesions in CAD patients.
\end{abstract}

Methods: From June 2010 to December 2011, a total of 170 patients (118 males (69.4\%); mean age, $63.9 \pm 12.1$ years) admitted with suspected CAD, were examined. Coronary angiography and IMT and PS in the carotid arteries ultrasound were obtained for all patients.

Results: The odds ratios related with the PS and the mean-CCA-IMT for the prediction of intermediate or high SYNTAX score were 1.13 (95\% Cl: 1.04-1.23; $p=0.003$ ) and 0.63 (95\% Cl: $0.02-22.29 ; p=N S)$, respectively. After adjustments for cardiovascular risk factors, genders, and age, PS only related to the presence of coronary arteries independently (odds ratio: $1.15 ; 95 \% \mathrm{Cl}: 1.06-1.24 ; \mathrm{p}<0.001$ ). To predict the intermediate or high SYNTAX score, the areas under the ROC curves for the PS and the mean-CCA-IMT were 0.698 (95\% Cl: $0.623-0.766 ; p<0.001)$ and $0.538(95 \% \mathrm{Cl}: 0.460-0.614 ; p=0.207)$, respectively. The cut-off value for the PS of 3.9 presented a negative predictive value of $92.4 \%$ and a positive predictive value of $30.8 \%$.

Conclusion: Carotid ultrasound parameters are capable of predicting the SYNTAX score. Furthermore, PS provided excellent negative predictive value for the complexity of coronary artery lesions.

Keywords: SYNTAX score; Plaque score; Intima-media thickness; Coronary artery disease

\section{Introduction}

Atherosclerosis is a systemic disease, with carotid and coronary arteries being the two most common atherosclerotic sites [1]. Carotid B-mode ultrasound can be used as a noninvasive method of examining the walls of peripheral arteries and for evaluating the intima-media thickness (IMT), presence of plaques [2,3]. In addition, carotid plaques are one of the major predictors of coronary artery multi-vessel disease with hypertension, old age, and increased IMT [1].

Numerous studies have been published the correlation between plaque score (PS), mean common carotid artery IMT (mean-CCAIMT) in carotid artery ultrasound findings, and the prevalence or complexity of coronary artery lesions in coronary artery disease (CAD) patients [4,5]. However, few studies have reported the association between the complexity of CAD and mean-CCA-IMT and PS [6]. The SYNTAX study also showed that the SYNTAX score (SXscore), which indicates the complexity of the lesion, is related to the prognosis of patients who underwent coronary revascularization [7].

Our study aimed to assess the strength of the correlation between carotid artery ultrasound parameters and the complexity of coronary artery lesions in CAD patients.

\section{Methods}

\section{Study population}

From June 2010 to December 2011, a total of 170 consecutive patients (118 males (69.4\%); mean age, $63.9 \pm 12.1$ years) were enrolled in the study of coronary angiography and carotid artery ultrasonography at the catheterization laboratory at Dongguk University Hospital (Gyeongju, Korea). The subjects are composed of 11 patients with stable angina, 73 cases of unstable angina, and 86 myocardial infarction patients. Patients who were hemodynamically unstable, had a poor quality of carotid imaging, or declined to participate, were excluded from the study. The baseline demographic, clinical and angiographic data were collected by trained researchers. Our study followed the Declaration of Helsinki and received written consent from all patients.

\section{Assessment of carotid ultrasound parameters}

The degree of carotid artery atherosclerosis was evaluated using mean-CCA-IMT and PS. The mean-CCA-IMT and the PS was evaluated by carotid-US (Acuson Sequoia c512, Siemens, Inc., United States). The ultrasonography of carotid arteries was performed as an ultrasound scanner with a $7.5 \mathrm{-MHz}$ linear probe by a single experienced sonographer. The sonographer and the interpreting 
cardiologist did not know the result of angiography. The patients were examined in the supine position with the head tilted backwards. After the carotid artery was placed by a transverse scan, the probe was rotated by $90^{\circ}$ to obtain a longitudinal image of the front and rear walls. The images of the far wall of the bilateral CCA, internal carotid arteries (ICA), and carotid bulbs, were in accordance to the recommendations of the American Society of Echocardiography Carotid Intima-Media Thickness Task Force [8]. The IMT was defined as the distance between the leading edge of the lumen-intima echo and the leading edge of the media-adventitia echo.

At least three measurements have been carried out for a distance of $1 \mathrm{~cm}$ in each of the distant walls of the CCA segments, the bilateral measurements were averaged to obtain the mean-IMT. If plaques were present in the segments used to measure the mean-IMT (Siemens, Syngo US Workplace), the plaque thickness was averaged into the mean-IMT measurement.

Focal intima-media thickening $\geq 0.9 \mathrm{~mm}$ specified the presence of a plaque. The PS was calculated by summing up the thickness of all plaques located in both carotid arteries.

\section{The Angiographic findings and SYNTAX score}

With the diagnostic angiogram, each coronary lesion producing $\geq$ $50 \%$ stenosis in blood vessels above $1.5 \mathrm{~mm}$ was scored individually; this score was added to provide the entire SXscore, which was calculated using the SXscore algorithm available on the SYNTAX website [9].

\section{Statistical analysis}

The data is expressed as the average \pm standard deviation or frequency (percentage). The patient characteristics between groups were compared with a t-test for continuous variables and a chi-square test for categorical variables. Logistic regression analysis was used to predict the presence of coronary artery disease, intermediate or high SXscore. Receiver-operating characteristic (ROC) curves were constructed to assess the best mean-IMT and PS values optimizing sensitivity to identify the presence of coronary artery disease or intermediate or high-SXscore patients. A $\mathrm{p}$ value $<0.05$ was considered statistically significant. SPSS ver.12 was used for the analyses.

\section{Results}

The patient's demographic characteristics were shown in Table 1. The median (SD) of age was $63.9(+12.1)$ years; $118(69.4 \%)$ subjects were male, 49 (28.8\%) had diabetes mellitus, 94 (55.3\%) had hypertension, and 94 (55.3\%) had current smoking. The median values of common carotid artery IMT (mean-CCA-IMT) and plaque score (PS) were $0.66 \pm 0.12 \mathrm{~mm}$ and $6.38 \pm 5.11 \mathrm{~mm}$, respectively. The patients of low (0-22), intermediate (23-32), and high (33 or more) SXscore numbered 133 (78.2\%), 27 (15.9\%), and 10 (5.9\%), respectively.

\begin{tabular}{|c|c|}
\hline Subject & $n=170$ \\
\hline Male (\%) & $118(69.4)$ \\
\hline Age (yrs) & $63.89 \pm 12.06$ \\
\hline BMI & $25.51 \pm 19.94$ \\
\hline Diabetes mellitus (\%) & $49(28.8)$ \\
\hline Hypertension (\%) & $94(55.3)$ \\
\hline Current smoking(\%) & $94(55.3)$ \\
\hline Hemoglobin A1c (\%) & $6.39 \pm 1.20$ \\
\hline Glucose (mg/dL) & $152.5 \pm 75.4$ \\
\hline $\mathrm{Cr}(\mathrm{mg} / \mathrm{dL})$ & $1.18 \pm 1.25$ \\
\hline Total cholesterol (mg/dL) & $182.8 \pm 46.4$ \\
\hline LDL cholesterol (mg/dL) & $116.3 \pm 45.0$ \\
\hline HDL cholesterol (mg/dL) & $45.4 \pm 13.1$ \\
\hline Triglyceride (mg/dL) & $134.2 \pm 72.6$ \\
\hline Mean CCA IMT (mm) & $0.66 \pm 0.12$ \\
\hline Plaque Score $(\mathrm{mm})$ & $6.38 \pm 5.11$ \\
\hline SYNTAX score & $15.2 \pm 9.4$ \\
\hline Low (0-22) (\%) & $133(78.2)$ \\
\hline Intermediate (23-32) (\%) & $27(15.9)$ \\
\hline
\end{tabular}


Citation: Jin-Wook Chung (2017) Association between Carotid Artery Plaque Score and SYNTAX Score in Coronary Artery Disease Patients. Gen Med (Los Angeles) 5: 301. doi:10.4172/2327-5146.1000301

Page 3 of 5

\begin{tabular}{|l|l|}
\hline High (33 or more) $(\%)$ & $10(5.9)$ \\
\hline STEMI $(\%)$ & $45(26.5)$ \\
\hline NSTEMI $(\%)$ & $41(24.1)$ \\
\hline U.A $(\%)$ & $73(42.9)$ \\
\hline S.A $(\%)$ & $11(6.5)$ \\
\hline
\end{tabular}

Table 1: Patient characteristics.

A significant correlation was seen between the SXscore and the mean-CCA-IMT (Spearman's rank correlation coefficient; $r=0.173$; $\mathrm{p}=0.024)$ (Figure 1), and the PS( $\mathrm{r}=0.233 ; \mathrm{p}=0.002$ ) (Figure 2). The odds ratios associated with the PS (1 each) and the mean-CCA-IMT $(0.1$ $\mathrm{mm}$ each) for the prediction of the intermediate or high SXscore were 1.13 (95\% CI: $1.04-1.23 ; \mathrm{p}=0.003$ ) and 0.63 (95\% CI: 0.02-22.29; $\mathrm{p}=0.798$ ), respectively. After adjustments for cardiovascular risk factors, genders, and age, PS only related to the presence of coronary arteries independently. (odds ratio: 1.15; 95\% CI: 1.06-1.24; $\mathrm{p}<0.001$ ) (Table 2).

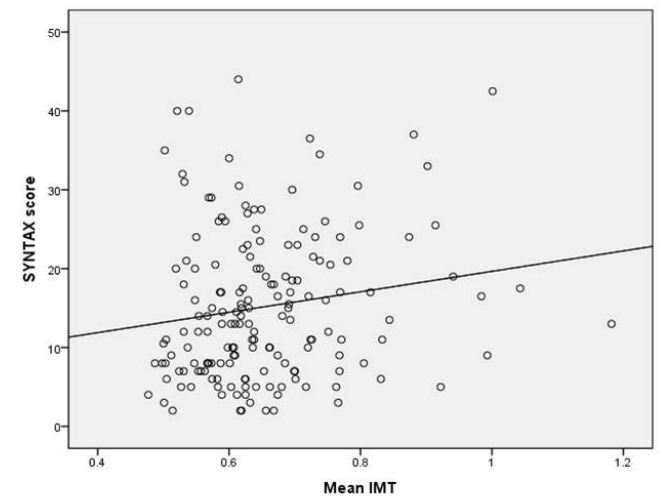

Figure 1: Correlation between the SYNTAX score and mean common carotid artery intima-media thickness (mean-CCA-IMT).

\begin{tabular}{|c|c|c|c|c|}
\hline Variable & $\begin{array}{l}\text { Unadjusted odds ratio, } \\
(95 \% \mathrm{Cl})\end{array}$ & P-value & $\begin{array}{l}\text { Adjusted odds ratio, } \\
(95 \% \mathrm{Cl})\end{array}$ & P-value \\
\hline Sex & $0.60(0.24-1.51)$ & 0.279 & - & \\
\hline Age & $1.02(0.97-1.07)$ & 0.432 & - & \\
\hline Diabetes mellitus & $2.69(1.15-6.29)$ & 0.023 & $2.69(1.21-5.98)$ & 0.015 \\
\hline Hypertension & $1.78(0.75-4.21)$ & 0.188 & - & \\
\hline Mean-IMT & $0.63(0.02-22.29)$ & 0.798 & - & \\
\hline Plaque score & $1.13(1.04-1.23)$ & 0.003 & $1.15(1.06-1.24)$ & $<0.001$ \\
\hline
\end{tabular}

Table 2: Predictors of patients with intermediate to high SYNTAX score. ROC curves for the PS and the mean-CCA-IMT were 0.698 (95\%CI: $0.623-0.766 ; \mathrm{p}<0.001)$ and 0.538 (95\% CI: $0.460-0.614 ; \mathrm{p}=0.207$ ), respectively (Figure 3 ). When cut-off value for mean-CCA-IMT was set to $0.8 \mathrm{~mm}$ to predict intermediate or high SXscore, the negative predictive value was $79.2 \%$, and the positive predictive value was $31.3 \%$. Similarly, the cut-off value for the PS of 3.9 presented a negative predictive value of $92.4 \%$ and a positive predictive value of $30.8 \%$.

Figure 2: Correlation between the SYNTAX score and plaque score (PS).

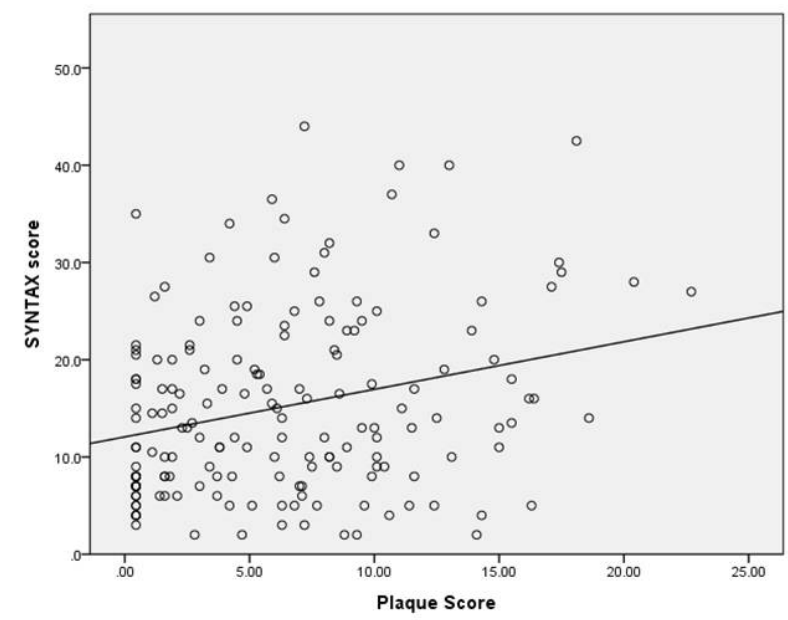

To predict the intermediate or high SXscore, the areas under the 


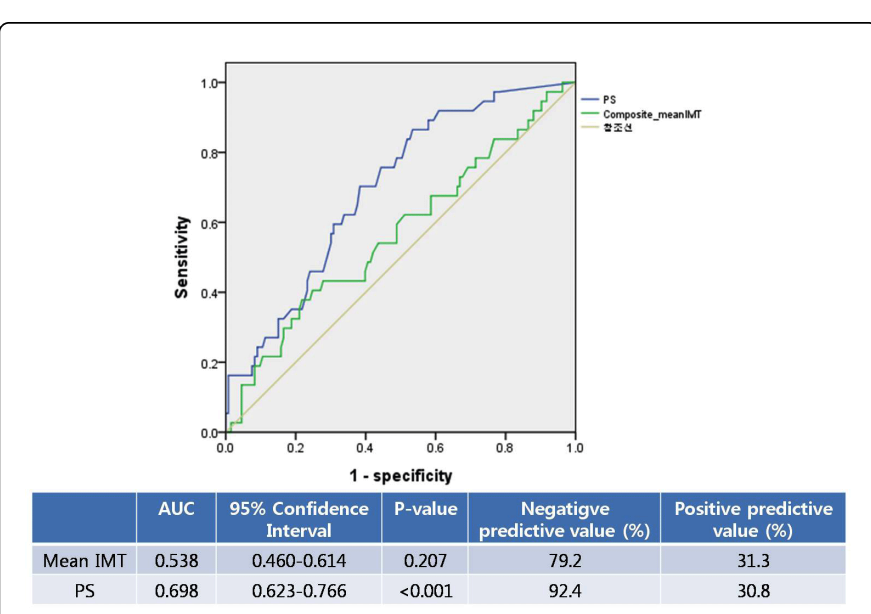

Figure 3: The ROC curve to identify patients with intermediate of high SYNTAX score.

\section{Discussion}

We have previously reported that all parameters of PS and IMT index (ultrasonography findings representing carotid atherosclerosis) are associated with cardiovascular risk factors. However, our study also shows that these indices are predictive of the severity of coronary artery lesions, which is now considered as one of the factors.

Numerous studies have investigated the relationship between ultrasonographic findings of carotid artery and CAD. Using the Bmode score, Craven et al. [10] found a strong independent association with CAD in patients over 50 years. Kallikazaros et al. [11] showed that carotid artery disease $(50 \%$ internal diameter stenosis $)$ was significantly associated with $\mathrm{CAD}$ in patients with chest pain. Rotterdam's study [12] revealed that carotid ultrasound findings were a new indicator of myocardial infarction after cardiovascular mortality in healthy adults over 55 years of age. Ogata et al. [13] investigated the relationship between IMT and the left main transthoracic calcification, and Sonoda et al. [14] presented their findings for coronary blood flow and IMT. Both studies reported a high IMT value to be a good indicator of the extent of CAD. In addition, Kablak-Ziembicka et al. [15] reported that IMT was significantly thicker in CAD-based groups, and their coronary angiographic findings indicated that IMT increased with increasing severity. Previous studies also showed that mean IMT increased with mean $\mathrm{CAD}$ increase, and patients with myocardial infarction had a significantly higher IMT than others. Thus, the high IMT observed in carotid ultrasound clearly shows a strong predictive value for CAD presence.

Other studies have also shown that PS is more important as a predictor of CAD than IMT.

Morito et al. [16] found that PS more closely represented the atherosclerotic status of the carotid artery than the IMT. They also found that subjects with carotid plaques in their study group had a higher risk of heart attack as compared to those with IMT. This indicated that the presence of carotid plaques is an independent predictor for the presence of CAD. Meta-analysis by Terzi et al. [17] showed a significantly higher diagnostic accuracy when PS predicted future myocardial infarction compared to IMT. However, the metaanalysis of Inabaa et al. [18] showed that although the diagnostic accuracy of PS was high,it was not significant compared to C-IMT for
CAD detection. Sakaguchi et al. [19] studied the relationship between coronary angiographic findings and IMT and PS, and concluded that both were indicators for the presence of CAD.

There are few published data on the relationship between PS and IMT (all ultrasonographic findings for carotid atherosclerosis) and the severity of CAD in patients with cardiovascular disease, and the correlation is somewhat weak [20]. Thus, the addition of carotid plaque measurements may extend the information regarding the status of coronary atherosclerosis. We therefore investigated the relationship between carotid IMT, PS and coronary artery lesions.

In our current study, given the complexity of lesions, we used SXscore as an independent risk factor for coronary artery multi-vessel disease in patients with carotid atherosclerosis. Our results suggest that the carotid plaque is more closely associated to coronary artery atherosclerosis. In this study, PS was also an independent predictor of the presence of CAD, but the mean IMT was not. Thus, PS values were better indicators than IMT for predicting the severity as well as the presence of CAD. Taken together, although the larger IMT is an important indicator of the early stages of atherosclerotic lesions and is a simple test method to assess the presence of CAD, our results show that higher PS values are more useful than IMT to predict the severity of CAD.

We set the cut-off values for mean-CCA-IMT (0.8 mm) and PS (3.9) to predict the presence of CAD, and intermediate or high SXscore in the ROC curve. Although these values appear too close to the normal value, this cut-off showed a good negative predictive value. Hence, we believe that the cut-off values used in our study are acceptable.

Our results suggest that the presence of a high PS value on carotid ultrasound has the greatest effect on determining the presence and severity of $\mathrm{CAD}$, and may be a decisive factor for future coronary angiography; with effective treatments such as statins or antihypertensive agents, it is possible to guarantee more active risk control.

There are some limitations to our results. This is not a populationbased study in which participants are randomly selected. Also, because the patients enrolled in our study were suspected to have ischemic heart disease, these subjects have a relatively higher risk than the healthy populations. Therefore, it is not clear whether our results are applicable to the general asymptomatic population (for example, at a health check-up). Obviously, a further study is necessary targeting general asymptomatic population and ideally should be confirmed prospective study in a large-scale study.

\section{Conclusion}

The carotid-US findings have a predictor for the SXscore. Furthermore, the PS and the mean-IMT provide excellent negative predictive values for the complexity of coronary artery lesions. Thus, large prospective studies will be needed to determine the link between these carotid-US findings and the complexity of coronary artery lesions.

\section{References}

1. Kwon TG, Kim KW, Park HW, Jeong JH, Kim KY, et al. (2009) Prevalence and significance of carotid plaques in patients with coronary atherosclerosis. Korean Circ J 39: 317-321. 
Citation: Jin-Wook Chung (2017) Association between Carotid Artery Plaque Score and SYNTAX Score in Coronary Artery Disease Patients. Gen Med (Los Angeles) 5: 301. doi:10.4172/2327-5146.1000301

Page 5 of 5

2. Belcaro G, Nicolaides AN, Laurora G (1996) Ultrasound morphology classification of the arterial wall and cardiovascular events in a 6-year follow-up study. Arterioscler Thromb Vasc Biol 16: 851-856.

3. Veller MG, Fisher CM, Nicolaides AN (1993) Measurement of the ultrasonic intima-media complex thickness in normal subjects. J Vasc Surg 17: $719-725$.

4. Rohani M, Jogestrand T, Ekberg M (2005) Interrelation between the extent of atherosclerosis in the thoracic aorta, carotid intima-media thickness and the extent of coronary artery disease. Atherosclerosis 179: 311-316.

5. O'Leary DH, Polak JF, Kronmal RA (1996) Thickening of the carotid wall: A marker for atherosclerosis in the elderly? Stroke 27: 224-231.

6. Ebrahim S, Papacosta O, Whincup P (1999) Carotid plaque, intima media thickness, cardiovascular risk factors, and prevalent cardiovascular disease in men and women: The British Regional Heart Study. Stroke 30: 841-850.

7. Serruys PW, Morice MC, Kappetein AP (2009) Percutaneous coronary intervention vs. coronary-artery bypass grafting for severe coronary artery disease. N Engl J Med 360: 961-972.

8. Stein JH, Korcarz CE, Hurst RT (2008) Use of carotid ultrasound to identify subclinical vascular disease and evaluate cardiovascular disease risk: A consensus statement from the American Society of Echocardiography Carotid Intima-Media Thickness Task Force. Endorsed by the Society for Vascular Medicine. J Am Soc Echocardiogr 21: 93-111.

9. Sianos G, Morel MA, Kappetein AP (2005) The SYNTAX Score: An angiographic tool grading the complexity of coronary artery disease. EuroIntervention 1: 219-227.

10. Craven TE, Ryu JE, Espeland MA (1990) Evaluation of the associations between carotid artery atherosclerosis and coronary artery stenosis. A Case-control study. Circulation 82: 1230-1242.

11. Kallikazaros I, Tsioufis C, Sideris S, Stefanadis C, Toutouzas P (1999) Carotid artery disease as a marker for the presence of severe coronary artery disease in patients evaluated for chest pain. Stroke 30: 1002-1007.
12. van der Meer IM, Bots ML, Hofman A, del Sol AI, van der Kuip DA, et al. (2004) Predictive value of noninvasive measures of atherosclerosis for incident myocardial infarction: The Rotterdam Study. Circulation 109: 1089-1094.

13. Ogata T, Yasaka M, Yamagishi M, Seguchi O, Nagatsuka K, et al. (2005) Atherosclerosis found on carotid ultrasonography is associated with atherosclerosis on coronary intravascular ultrasonography. J Ultrasound Med 24: 469-474.

14. Sonoda M, Yonekura K, Yokoyama I, Takenaka K, Nagai R, et al. (2004) Common carotid intima-media thickness is correlated with myocardial flow reserve in patients with coronary artery disease: A useful noninvasive indicator of coronary atherosclerosis. Int J Cardiol 93: 131-136.

15. Kablak-Ziembicka A, Tracz W, Przewlocki T, Pieniazek P, Sokolowski A, et al. (2004) Association of increased carotid intima-media thickness with the extent of coronary artery disease. Heart 90: 1286-1290.

16. Morito N, Inoue Y, Urata M (2008) Increased carotid artery plaque score is an independent predictor of the presence and severity of coronary artery disease. J Cardiol 51: 25-32.

17. Terzi S, Sayar N, Bilsel T (2007) Tissue Doppler imaging adds incremental value in predicting exercise capacity in patients with congestive heart failure. Heart Vessels 22: 237-244.

18. Inabaa Y, Chen JA, Bergmannb SR (2012) Carotid plaque, compared with carotid intima-media thickness, more accurately predicts coronary artery disease events: A meta-analysis. Atherosclerosis 220: 128-133.

19. Sakaguchi M, Kitagawa K, Nagai Y (2003) Equivalence of plaque score and intima-media thickness of carotid ultrasonography for predicting severe coronary artery lesion. Ultrasound Med Biol 29: 367-371.

20. Adams MR, Nakagomi A, Keech A (1995) Carotid intima-media thickness is only weakly correlated with the extent and severity of coronary artery disease. Circulation 92: 2127-2134. 